\title{
The Development of Polysemous 'One’-phrase in Mandarin Chinese
}

\author{
I-Hsuan Chen*
}

LSA Annual Meeting, Minneapolis, January 2-5, 2014

1. Introduction. Mandarin Chinese has a salient typological feature in the occurrence of classifiers. Studies on numeral-classifier phrases in Mandarin Chinese have garnered much attention (Chao 1968, Cheng \& Sybesma 1999, Zhang 2013). However, the functions other than counting have not received due attention. Among the Mandarin numeral phrases, the 'one'phrases exhibit the most robust contextualized readings. The numeral 'one' is neither simply a number, nor a classifier merely for classifying a noun. When incorporated into the 'one'classifier-noun sequence, such as yi-li-mi 'a grain of rice', yi-dian-shui 'a dot of water', and yipian-shuye 'a piece of leaf', their meanings range from maximality (wholeness), to minimality (zero), showing synchronic variation. These synchronic variants are often treated separately, deprived of a holistic perspective, but they are actually closely related when examined through a historical lens. With historically oriented contextual analysis, this study adopts a constructional approach (Croft \& Cruse 2004, Goldberg 2006, Traugott \& Trousdale 2013) to analyze the grammaticalization of 'one'-phrases, providing a more precise way to study both synchronic discrepancy and diachronic development.

2. Synchronic variations of 'one'-phrases. Modern Mandarin 'one'-phrases participate in a number of different constructions: counting 'one', downtoner 'a little bit/some', minimality, maximality. The typical function of a 'one'-phrase is for counting, as the Mandarin classifiers are claimed to individualize the denotation of nouns for the purpose of counting and measuring (Chao 1968, Cheng \& Sybesma 1999), as shown in (1) ${ }^{1}$. The contrast between the numerals, 'one' and 'two', shows yi 'one' in its cardinal function.

$\begin{array}{lllllll}\text { (1) wo jian-dao } & \text { [yi pian } & \text { shuye], bu } & \text { shi liang } & \text { pian } \\ \text { I picked one CL_piece } & \text { leaf } & \text { not } & \text { copular } & \text { two } & \text { CL } \\ \text { 'I picked up one leaf, not two.' } & & & & & \end{array}$

However, yi 'one' does not always behave as a typical numeral, as in (2), where 'one' cannot be replaced by other cardinals. Mandarin 'one'-phrases literally refer to an individual item, hence they are often associated with a small amount. Crosslinguistically, expressions for a smallest amount, such as English budge an inch and blink a wink, tend to become emphasizers in negative sentences, and therefore they have been termed negative polarity items (NPIs) (Israel 2011). The 'one'-phrase in (2) functions as a minimizer NPI, whose minimal amount enables one to order and compare different possibilities, evoking a process called scalar reasoning, a basic cognitive process in human beings.

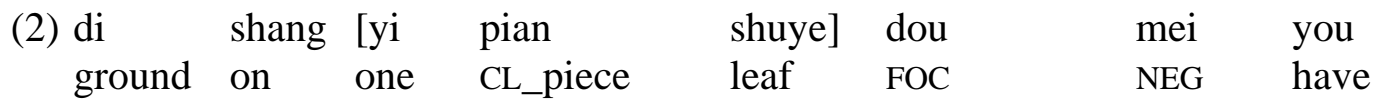

'There is no leaf on the ground.'

The 'one'-phrase in (3) reveals a meaning of 'wholeness'. This numeral 'one' leads to the 'all over' interpretation. It is treated as a determinative quantifier or a non-numeral 'one' with a maximal meaning (Zhang 2013).

*Author: I-Hsuan Chen, University of California, Berkeley (ihsuanchen@berkeley.edu).

${ }^{1}$ Abbreviations used in this proposal include: CL: classifier, FOC: focus, NEG: negation, NUM: numeral. 


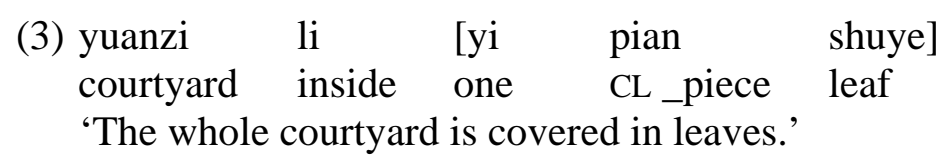

The 'one'-phrase can also refer to an indefinite small amount as English a little, as in (4). In this case, the 'one'-phrase is used to suggest a small amount.

$\begin{array}{clllll}\text { (4) yong } & \text { [yi } & \text { dian } & \text { yezi] } & \text { zhuangshi } & \text { zhe dao cai } \\ \text { use } & \text { one } & \text { dot } & \text { leaf } & \text { garnish } & \text { this }\end{array}$

'Use a little bit of leaves to garnish this dish.'

As the examples suggest, the interpretations of 'one'-phrases are constrained by the context. It is not merely the reading of 'one' but also the properties of classifiers and the contexts where 'one'-phrases dwell that influence the reading of 'one'-phrases. In any case, the distinct functions of Mandarin 'one'-phrases are not unrelated; rather, they are reflections of different stages of semantic development. For instance, Mandarin dian 'dot' first appeared as a noun referring specifically to an ink dot in Old Chinese. Its zero-dimensionality was employed in poetry to describe a distant round shape, such as yi dian qing yang 'one dot of sun' and yi dian bai ou 'one dot of seagull' in Middle Chinese. The constraint for the round shape in yi dian 'one dot' faded away in Early Mandarin. Yi dian 'one dot' came to be used to describe abstract nouns, such as niantou 'thought' and feng 'wind' to emphasize the small quantity. The property of indefiniteness in the 'one dot'-phrases became prominent in Early Mandarin and employed to mean 'some/a little bit'. In modern Chinese, while all the usages are still current, the phrases have developed as NPIs emphasizing the degree of negation, as in (5).

(5) ta $[y i$ dian xinxin] dou mei you he one dot confidence FOC NEG have 'He doesn't have any confidence at all.'

The sequence ‘one'-CL-NOUN has developed multiple functions over time. A later developed function can be considered as a construction with its syntax, semantics, and pragmatics encoded.

3. Diachronic development behind polysemies in 'one'-phrases. The synchronic variation of the 'one'-phrases is associated with their diachronic development. Typically, previous studies of Mandarin numeral phrases have focused mainly on their structural aspects. To pin down the actual interpretations of the 'one'-phrases, however, it is necessary to consider their semantics and pragmatics as well as their constructions in interaction.

3.1 DATA SOURCE. The data are gathered from the Chinese corpora of Academia Sinica, containing a complete collection of Chinese texts from different periods. The 'one'-phrases are examined in the three subcorpora: Old Chinese (8c. - 1c. B.C.), Middle Chinese (1c - 6c. A.D.) and Early Modern Mandarin (7c. - 19c A.D.). These corpora contain materials from diverse genres. The various sources provide data on all the distinct usages of the 'one'-phrases, making it possible to put together a clear picture of how contexts shape interpretations.

3.1 CONSTRUCTIONAL CHANGE. The 'one'-phrases when understood in different readings have different syntactic, semantic, and pragmatic properties that are interdependent on each other. The sequence, 'one'-CL-NOUN, can be viewed as having relatively indeterminate meaning and it acquires its meaning from the constructions it appears in. The pairing of form and meaning is conditioned by related constructions. Chinese classifiers first appeared in Old Chinese. It was in Middle Chinese that the pattern, 'one'-CL-NOUN, became a pervasive counting phrase. The 
minimizer NPI function occurred in late Old Chinese when the sequence, 'one'-CL-NOUN, appeared with negation. The 'one'-phrases as minimizer NPIs were further associated with the quantifiers which also served as focus markers in Early Mandarin. The negation and focus constructions turn the 'one'-phrase in (6) into an NPI. The focus marker in (7) is a quantifier exhausting all the possibilities in seeing people. The 'one'-phrase represents the least possibility. Negating the minimal amount pragmatically leads to total negation. The combination of the focal construction and the 'one'-phrases reflects the emphatic function encoded in minimizer NPIs.

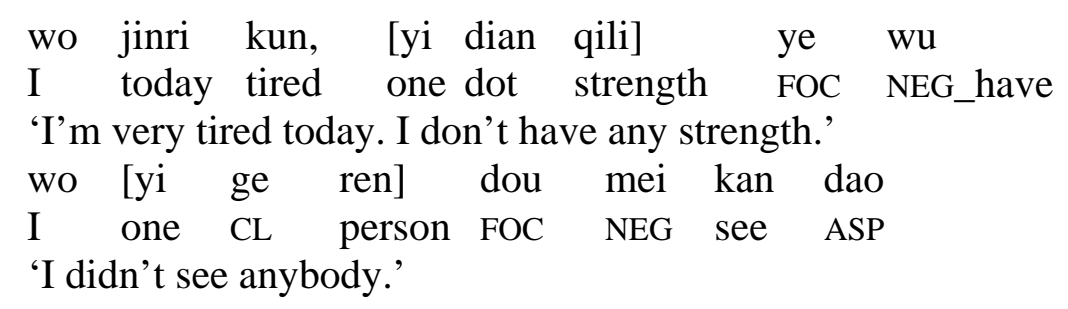

The smallness of quantity entailed in 'one'-phrases has another path of development. As in (8), the 'one'-phrase means an indefinite amount, similar to English 'some' and 'a little'. In this case, the indefiniteness is weighted over smallness when compared to the function of a minimizer NPI.

(8) chuanjia mai [yi dian chai mi]

fisherman buy one dot wood rice

'The fisherman bought some wood and rice.'

Besides minimality, some of the 'one'-phrases have also developed a reading of maximality. For instance, the sequence, 'one'-pian 'piece'-NOUN, can refer to maximality in addition to counting. In Old Chinese, the phrase was used for counting thin flat objects, as in (9a). The 'one'-phrase extended to refer to a covered area as in (9b) in Middle Chinese. In Early Mandarin, the 'one'phrase could take an abstract noun to mean 'wholeness' or 'all over the...', as in (10). The classifiers in the 'one'-phrases with a maximal reading are named temporary classifiers, such as bizi 'nose' and shen 'body' or some specific classifiers such as pian 'piece' and tuan 'round mass' (Chao 1968). The reading wholeness does not appear if the occurring numeral is not 'one'.

(9) a. yi pian hei shi b. $\begin{aligned} & \text { yi pian huo qi } \\ & \text { one piece black stone } \\ & \text { 'a piece of black stone' }\end{aligned}$
'... fire breaking out all over'
(10) yi lian youchou
one face worry
'...the face looked all worried...'

The conditions for developing the reading maximality are the occurrence with gradable predicates, such as lin 'to wet', sa 'to sprinkle', $t u$ 'smear' and adjectives referring to wholeness, zheng 'whole', or completion, as in (11).

(11) tu yi zheng lian niba
smear one whole face mud
'smear mud all over the face'

The multiple readings of 'one'-phrases share one feature: scalar implication. The 'one'-phrase serves as a reference point on a scale, invoking different scalar inferences when situated in different scale backgrounds. On a counting scale, the 'one'-phrase refers to a unit. When behaving as a minimizer NPI, the 'one'-phrase indicates the bottom of a scale, whereas it refers 
to the top of a scale for maximality. The scalar implications are built into the whole construction instead of one lexeme through grammaticalization. Each function of the 'one'-phrase is treated as a construction that has different relationships among its three components with its scalarity encoded. Different parts of semantics are emphasized in different readings. The various readings of the 'one'-phrases reflect incremental development across stages, as shown in Figure $1^{2}$.

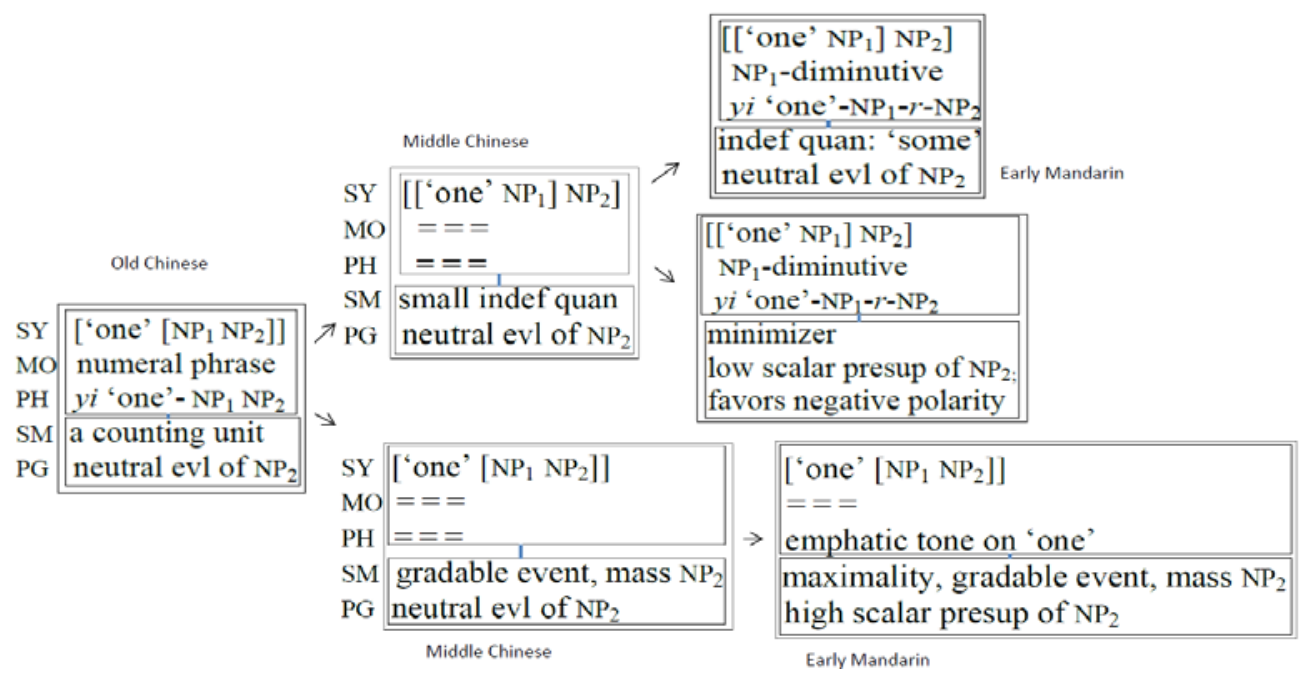

Figure 1. The constructional development of 'one'-phrases

4. Diachronic development underlying synchronic variation. The incremental development of 'one'-phrases can be treated as the accumulation of a series of local steps formed through reanalysis of syntactic structures, resulting in new configurations from old materials. The changes are well constrained by their conjoined constructions. The constructional account shows that it is the entire construction, rather than a single lexical item, the numeral or the classifier, that has undergone grammaticalization.

\section{References}

Chao, Yuen-Ren. 1968. A Grammar of Spoken Chinese. Berkeley: University of California Press.

Cheng, Lisa Lai-Shen and Rint Sybesma. 1999. Bare and not-so-bare nouns and the structure of NP. Linguistics Inquiry 30: 509-542.

Cheng, Lisa Lai-Shen \& Anastasia Giannakidou. 2012. The Non-Uniformity of wh-indeterminates with free choice and polarity in Chinese. In Kook-hee Gil, Stephen Harlow \& George Tsoulas, (eds.), The Nature of Quantification and Crosslinguistic variation. 123-154. Oxford: Oxford University Press.

Croft, William \& D. Alan Cruse. 2004. Cognitive Linguistics. Cambridge: Cambridge University Press.

Fried, Mirjam \& Jan-Ola Östman. 2004. Construction grammar: A thumbnail sketch. In Fried Mirjam \& Jan-Ola Östman (eds.), Construction grammar in a cross-language perspective. 11-86. Amsterdam/Philadelphia: John Benjamins Publishing Company.

Goldberg, Adele E. 2006. Constructions at work: The nature of generalization in language. Oxford: Oxford University Press.

Israel, Michael. 2011. The Grammar of Polarity: Pragmatics, Sensitivity, and the Logic of Scales. Cambridge: Cambridge University Press.

Traugott, Elizabeth Closs \& Graeme Trousdale. 2013. Constructionalization and Constructional Changes. Oxford: Oxford University Press.

Zhang, Ning. 2013. Classifier Structures in Mandarin Chinese. Berlin: Mouton De Gruyter.

\footnotetext{
${ }^{2}$ Notations used in this figure are: SY: syntax, MO: morphology, PH: phonology, SM: semantics, PG: pragmatics, '=
} =’: 'carries over from prior stage', 'evl': evaluation, indef: indefinite, presup: presupposition, quan: quantity. 